17-1 | 2015

Éclat

\title{
Enquête sur les conditions de travail auprès de professeurs d'éducation physique et sportive et perspectives d'action
}

Survey of the Working Conditions of Physical Education and Sport Teachers and Prospects for Action

Encuesta de condiciones de trabajo de profesores de educación física y deportes y perspectivas de acción

Fabien Coutarel, Céline Mardon, Serge Volkoff, Jacques Fiard, Michel Récopé, Joffrey Beaujouan et Géraldine Rix

\section{CpenEdition}

Journals

Édition électronique

URL : http://journals.openedition.org/pistes/4391

DOI : $10.4000 /$ pistes. 4391

ISSN : 1481-9384

Éditeur

Les Amis de PISTES

Référence électronique

Fabien Coutarel, Céline Mardon, Serge Volkoff, Jacques Fiard, Michel Récopé, Joffrey Beaujouan et Géraldine Rix, «Enquête sur les conditions de travail auprès de professeurs d'éducation physique et sportive et perspectives d'action », Perspectives interdisciplinaires sur le travail et la santé [En ligne], 17-1 | 2015, mis en ligne le 31 mars 2015, consulté le 19 avril 2019. URL : http://

journals.openedition.org/pistes/4391; DOI : 10.4000/pistes.4391

Ce document a été généré automatiquement le 19 avril 2019.

Pistes est mis à disposition selon les termes de la licence Creative Commons Attribution - Pas d'Utilisation Commerciale - Pas de Modification 4.0 International. 


\title{
Enquête sur les conditions de travail auprès de professeurs d'éducation physique et sportive et perspectives d'action
}

\author{
Survey of the Working Conditions of Physical Education and Sport Teachers and \\ Prospects for Action \\ Encuesta de condiciones de trabajo de profesores de educación física y deportes y \\ perspectivas de acción
}

Fabien Coutarel, Céline Mardon, Serge Volkoff, Jacques Fiard, Michel Récopé, Joffrey Beaujouan et Géraldine Rix

\section{Le travail enseignant et l'ergonomie}

1 Lorsque la question de la santé est posée aux intervenants du monde de l'éducation, la pensée commune renvoie spontanément à la santé de l'élève. Le système éducatif constitue un champ d'investigations et de recherches au centre duquel l'élève a toujours focalisé l'attention. Les deux autres pôles du «triangle pédagogique » (Houssaye, 2000), le savoir et l'enseignant, font également l'objet de nombreux travaux visant à améliorer la compréhension des processus d'apprentissage. L'enseignant est concerné au titre de sa contribution à la construction de l'activité de l'élève. Ainsi, nous pourrons trouver en sciences de l'éducation des travaux mobilisant l'analyse de l'activité de situations d'apprentissages (par exemple Lipp et Ria, 2012). Cependant, les situations de travail dont le rapport à l'élève est lointain restent ainsi peu étudiées dans ces travaux.

2 Selon Lancry-Hoestlandt (2013), la première apparition du mot «ergonomie » dans un arrêté du ministère de l'Éducation nationale date de 1977 ; il est proposé au Département de la recherche médicale de l'Institut National de Recherche Pédagogique de travailler sur « l'analyse des caractéristiques des postes de travail de l'élève en situation d'étude ou 
d'apprentissage sous l'angle de l'ergonomie scolaire ». Lancry-Hoestland nous rappelle la définition de l'ergonomie scolaire, universitaire et éducative proposée par Lambert et Pascal (1983) :

«Une méthode d'action qui se propose d'optimiser l'ensemble du processus éducatif. Elle fait appel nécessairement à la biologie humaine et à la psychologie dans une perspective anthropologique. Englobant l'ensemble des facteurs du processus éducatif, son objet est constitué à la fois par les rapports au travail de l'élève et de l'enseignant, par l'équipement et le matériel utilisé, par le milieu, mais aussi par les conditions temporelles et sociales dans lesquelles les projets éducatifs se déroulent. »

3 L'approche systémique de l'activité de l'enseignant à travers l'ensemble des déterminants des situations diverses et variables qu'il gère, prenant en compte la diversité des contextes des situations de travail (Delvolvé et Margot, 2001 ; Lancry-Hoestlandt, 2013), y compris les situations de travail en amont et en aval du face à face avec l'élève, continue de caractériser la perspective ergonomique, notamment au sein du courant de l'ergonomie de l'activité (Daniellou et Rabardel, 2005). Le rapport à l'élève n'est pas évacué de cette approche : la qualité des apprentissages des élèves est indissociablement liée à la dynamique de construction de l'expérience professionnelle où les conditions du travail enseignant s'avèrent déterminantes, notamment dans des situations où la présence de l'élève est lointaine. Par contre, l'analyse du travail de l'enseignant n'y est pas systématiquement subordonnée à la question première de l'apprentissage des élèves, et les autres enjeux de l'activité enseignante y ont toute leur place.

Enfin, dans cette perspective, la question de la santé et du développement du professionnel devient incontournable pour la réflexion sur sa performance. Nous pouvons ainsi situer les travaux qui s'inscrivent dans une perspective d'action à travers la formation (Teiger et Montreuil, 1995), où l'analyse ergonomique du travail est première (Montmollin, 1974; Lacomblez et coll., 2007). La formation des adultes est une préoccupation forte de l'ergonomie de l'activité depuis de nombreuses années (Delvolvé et Margot, 2001; Teiger et Lacomblez, 2006; Falzon et Teiger, 2011). En contribuant au développement des compétences du formateur et à l'efficacité des formations, l'ergonomie contribue à la construction de la santé. Nous pouvons par exemple nous référer à De Montmollin (1993), qui définit la « santé cognitive " par le développement de compétences, ou encore à tous les travaux à orientation psychologique qui établissent des liens entre santé mentale et reconnaissance, sens du travail, perception du travail bien fait.

Dans le système éducatif, l'enseignant d'Éducation Physique et Sportive (EPS) occupe une place particulière au sein du corps enseignant : il se distingue fortement de ses collègues d'autres disciplines en situation d'enseignement par l'espace investi, les formes de sollicitations des élèves, les risques encourus pour les élèves et pour lui-même, etc. On ne peut donc se résoudre à désigner un «métier d'enseignant » homogène, quels que soient les contextes, les niveaux et les disciplines. De la maternelle à l'université, les lieux sont diversifiés, les caractéristiques des élèves sont variées et variables, tout comme le rapport social et culturel aux différentes disciplines. La prise en compte de la variabilité des contextes professionnels est indispensable pour produire des analyses précises et circonstanciées à même de servir des perspectives d'amélioration des situations concernées. Au-delà d'un cadre commun, il y a donc des dissemblances entre le métier de professeur d'atelier, d'enseignant de classe maternelle et d'enseignant d'EPS. 


\section{L'enseignant d'EPS : un travailleur « comme les autres »?}

6 Les sciences humaines et sociales ont permis d'élaborer progressivement des approches enrichies du travail. Tout travail suppose une mobilisation physique, cognitive et subjective de celui ou celle qui l'exerce en lien avec d'autres dans un cadre partiellement contraint, mobilisation que les ergonomes appellent « activité de travail ».

7 L'ergonomie de l'activité développe un point de vue spécifique: une approche systémique, intégrant les dimensions subjectives, physiques, collectives, sociales, organisationnelles, culturelles (Guérin et coll., 1997 ; Falzon, 2004 ; Daniellou et Rabardel, 2005). La prise en compte de ces dimensions hétérogènes du travail situe de manière centrale la question des débats de normes et des injonctions contradictoires (Schwartz, 1994 ; Coutarel, 2011).

8 Par exemple : assurer la qualité d'apprentissages individualisés tout en recevant de plus en plus d'élèves en classe, quelquefois en contradiction avec les textes sur la sécurité (en natation notamment); dispenser des contenus différenciés à des élèves aux ressources très hétérogènes (classes mélangées par le jeu des options ou des «menus » offerts au choix des élèves); veiller à la sécurité des élèves alors que la conception des installations et espaces de travail n'a pas intégré cette dimension (consignes d'interdictions formelles, telle celle de se suspendre aux panneaux de basket, aux cages de handball); être rigoureux sur la discipline alors que l'administration de l'établissement est négativement évaluée si elle exclut trop d'élèves, etc.

9 Les processus de construction ou d'atteinte à la santé des travailleurs semblent largement marqués par les conditions dans lesquelles les injonctions peuvent être traitées, débattues et gérées au sein des organisations (Coutarel, 2011). L'intensification générale du travail (Davezies, 1999 ; Askenazy et coll., 2006) qui contribue à renforcer et multiplier les contradictions de ces injonctions au sein des organisations concerne-t-elle le système éducatif?

10 Les enseignants d'EPS restent la cible de représentations sociales dominantes qui ne favorisent pas l'interrogation de cette pratique professionnelle en tant que travail.

11 La pratique sportive étant perçue comme une détente, il est commun de penser que le travail consistant à l'enseigner s'en rapproche. La "profession sportive » est à ce point idéalisée, voire entretenue en ces termes par les acteurs eux-mêmes, dans l'imagerie populaire, que ses avatars (vieillissement, fatigue, usure, douleurs articulaires...) sont régulièrement cachés, voire réfutés. On en oublierait presque que le travail sportif est un « vrai » travail (Fiard et coll., 2008).

12 On constate cependant que des facteurs de risques d'atteintes à la santé au travail identifiés et largement décrits dans la littérature scientifique à propos des troubles musculo-squelettiques ou des risques psychosociaux au travail, pour d'autres populations de travailleurs (enseignants ou autres) semblent également présents dans les situations professionnelles quotidiennes des enseignants d'EPS. On peut citer les «nuisances classiques » : par exemple une station debout permanente; des déplacements nombreux tout au long de la journée; l'adoption de postures contraignantes lorsqu'il s'agit de démontrer - le plus souvent sans échauffement préalable ; des conditions d'exposition au bruit dans des salles mal insonorisées et quelquefois encore amiantées, au froid l'hiver, à 
la chaleur des piscines, à la poussière des gymnases... Par ailleurs, les discours tendent à souligner des difficultés liées à l'estime de soi, à la reconnaissance, au soutien. Enfin, audelà de la difficulté d'assurer une attitude active constante, de démontrer, de se confronter aux élèves dans les situations de jeu, il s'agit aussi d'affronter les conduites parfois agressives des élèves ou tout simplement leur passivité. On évoque peu, pour cette profession, la difficulté de "vieillir bien dans son travail ». Cau-Bareille (2009) a déjà souligné cet enjeu de fin de carrière pour le métier d'enseignant de manière générale.

Quelques travaux portant spécifiquement sur le travail des enseignants ont déjà pu asseoir ce constat : au-delà de l'image populaire d'un métier idéalisé, à l'abri de la fatigue et de l'usure, on repère dans les discours des acteurs les multiples aspects d'une intensification des pressions dans le cadre scolaire qui viennent se surajouter aux conditions écologiques d'exercice (Thomas-Bion et Fiard, 2008). La fatigue quotidienne et l'usure professionnelle des enseignants exerçant en collège constituent une vraie épreuve (Van Zanten, 1997). Les problèmes de discipline liés aux incivilités et au désordre dans les classes (Thin, 2002) occasionnent fatigue et usure (Carraud, 2006 ; Gal-Petitfaux et Saury, 2002; Vors et Gal-Petitfaux, 2007). Un regard de plus en plus institutionnel et administratif et plus scrutateur, une présence parentale plus insistante font que l'accident d'élève, une chute imprévue, une blessure, donnent plus qu'avant lieu à éclaircissement, enquête, ouvrant quelquefois sur la voie judiciaire. Thomas-Bion et Fiard (2010) ont montré le souci permanent, quelquefois obsessionnel, de ces enseignants d'échapper à l'accident d'élèves, quitte à abaisser la demande énergétique des pratiques, voire à les transformer pour les rendre moins dangereuses, délaissant des pans entiers des pratiques sportives (par exemple les lancers athlétiques, les pratiques acrobatiques, les sports collectifs de grand terrain). Désormais les règles de sécurité se doivent d'être scrupuleusement respectées, les systèmes de protection systématiquement installés, les activités judicieusement choisies. Tous ces éléments semblent pouvoir constituer des motifs d'une angoisse de l'imprévu, en même temps qu'une démotivation partagée entre l'enseignant et ses élèves pour des pratiques de moindre intérêt.

Enfin une raison démographique : la hausse continue de l'âge moyen des enseignants du second degré (43,1 ans aujourd'hui) est due à la fois à la diminution des enseignants de moins de 30 ans (chute des postes ouverts durant les années 2000 au concours de recrutement) et à l'augmentation de la durée d'exercice. Statistiquement, la population des enseignants d'EPS vieillit et rien, à notre connaissance, n'est prévu pour prévenir ou parer les conséquences de cette tendance.

\section{Méthodologie de l'enquête auprès d'enseignants d'EPS et d'analyse des résultats}

15 Cette enquête a été conduite à l'initiative de l'équipe de chercheurs, sans demande préalable au sens de l'ergonomie ${ }^{1}$. Dans l'équipe, deux chercheurs sont très impliqués dans la formation des enseignants d'EPS depuis plusieurs dizaines d'années. Ils ont eu l'occasion de former aux concours d'entrée dans le métier, d'accompagner les premières années de pratique, de concevoir et d'animer des stages de formation continue à destination des enseignants d'EPS. Le sentiment d'une difficulté croissante à vivre le métier, notamment les dernières années de carrière, s'est progressivement construit chez eux. Ils sont à l'initiative de cette enquête visant à objectiver et comprendre cette impression d'experts. L'approche ergonomique a contribué à inspirer les questions, mais 
la contribution des ergonomes s'est essentiellement concentrée sur l'analyse et l'interprétation des résultats, ainsi que sur la discussion autour de perspectives d'action.

L'enquête présentée ici vise précisément à explorer les conditions et les conséquences de la mise en péril ou de la construction de sa santé par le travail pour les professeurs d'EPS de collège et de lycée. Elle a été conduite à l'occasion d'un stage académique de formation continue en région Auvergne (France), auprès des professeurs d'EPS participants. Le questionnaire était distribué à l'un des stands où deux des chercheurs de l'équipe se tenaient à disposition pour répondre à d'éventuelles questions, les répondants étant invités à renseigner le questionnaire immédiatement après sa distribution.

17 Le cadre théorique présenté ci-dessus a orienté la constitution du questionnaire qui se structure autour de quatre parties ${ }^{2}$.

18 La première se donne pour objet l'investigation des conditions d'exercice des enseignants d'EPS. Que décrivent-ils de leur relation à la classe, du temps passé au travail, de la relation avec les parents, des possibles conséquences des accidents en cours, du soutien de l'administration en cas de conflit avec un élève, des charges administratives, etc. ?

19 La seconde partie du questionnaire se propose de collecter les problèmes de santé ressentis et le vécu du travail. En d'autres termes : comment les enseignants disent-ils leur santé et quelle image pensent-ils renvoyer à leurs élèves? Pour ces deux premiers volets, trois codages des réponses cadrent les propositions: l'axe de la tolérabilité (de " parfaitement admissible » à « tout à fait intolérable »), l'axe diachronique (de " cela va mieux que par le passé » à "cela devient pire»), enfin l'axe de la fatigabilité (de « aucunement fatigant » à « épuisant »).

Dans la troisième partie sont proposés aux enseignants des scénarii différents quant à la possibilité d'un changement de cadre, d'une sortie du métier.

21 Enfin, la quatrième partie concerne les valeurs, le rapport au travail : quelle crédibilité accordent-ils à leur profession? Quel est leur sentiment à propos des effets que leur métier produit dans les conditions d'exercice qui sont les leurs? Conseilleraient-ils leur profession à un proche en âge de s'y engager? Quelle fut pour eux-mêmes, si tant est que l'on puisse accorder crédit au concept, leur « vocation »?

22 Le questionnaire comportait donc un certain nombre de questions sous forme d'axes, avec des modalités de réponses allant de 1 (réponse souvent la plus "positive») à 7 (réponse la plus «négative »). Les résultats décrits ci-après sur ces questions sont issus d'une dichotomisation de ces réponses, en opposant les réponses 5 et plus aux autres modalités possibles.

23 La population ayant répondu au questionnaire est constituée de 218 professeurs d'EPS, avec une répartition équilibrée par genre (108 hommes et 110 femmes) et un âge médian de 42 ans. Ces 218 réponses exploitables correspondent à $54 \%$ des 405 enseignants inscrits à la formation et à $37 \%$ des 591 enseignants de l'académie.

24 Le mode de recueil et les taux de répondants n'assurent pas la représentativité de notre échantillon. C'est pourquoi dans la présentation de nos résultats nous nous attarderons peu sur la fréquence d'ensemble de telle modalité de réponse. Nous donnerons ces fréquences à titre indicatif, puis nous privilégierons les comparaisons entre souspopulations, et une analyse typologique.

Dans ce but, après un tri à plat (description des réponses pour chaque variable), nous avons réalisé des tris croisés par sexe et par âge. Ces premiers résultats quantitatifs 
associés à des hypothèses issues de l'expérience de terrain des chercheurs ont aidé au commentaire d'une analyse des correspondances multiples (ACM) réalisée par la suite. Des régressions multivariées (modèle logistique) ont enfin permis d'investiguer les relations entre travail et santé dégagées par l'ACM.

\section{Résultats}

Les résultats présentés ci-après vont des plus généraux aux plus spécifiques : appréciation générale du métier; difficultés liées aux élèves; difficultés liées au collectif et à l'organisation du travail ; difficultés relatives à l'institution scolaire et au statut social ; les différences liées au sexe; les différences liées à l'âge ; l'association des difficultés à des problèmes d'organisation du travail, de charge de travail et de sens du travail; les relations santé-travail.

\subsection{Une appréciation générale plutôt positive des enseignants d'EPS sur leur métier}

On peut distinguer deux catégories dans les résultats obtenus : ceux qui relèvent d'une perception générale de l'activité professionnelle et ceux qui concernent des questions plus spécifiques à certaines dimensions de cette activité. Les résultats obtenus peuvent sembler contradictoires.

Les professeurs d'EPS se jugent globalement en bonne forme (160 sur 218, soit $73 \%$ d'entre eux) et préservés en matière de conditions de travail. Seulement 13 d'entre eux se disent en mauvaise condition physique et "usés». On recueille 47 arrêts de travail déclarés au cours des six derniers mois, toutes causes confondues.

Ils pensent renvoyer une image positive à leurs élèves (186 se notent au-dessus de 6/10, 156 se notent à 7 et plus). Par ailleurs, 198 des 218 répondants croient aux bienfaits de l'EPS en primaire, 183 en collège, 139 en lycée. Ils sont tout autant persuadés (à $85 \%$ ) des effets bénéfiques de l'EPS qu'ils enseignent, dans les conditions d'exercice qui sont les leurs.

Enfin, ils revendiquent à une forte majorité la vocation qui les a engagés dans le métier (186 sur 218) et fort nombreux sont celles et ceux qui la revendiquent encore (151), même si un tiers d'entre eux exprime de concert des formes de désillusion, de lassitude et de stress.

31 Cependant, les réponses aux questions plus précises laissent apparaitre un paysage plus contrasté. Relativement à la santé, on peut retenir les principaux résultats suivants :

- 115 enseignants sur 218, soit plus de la moitié des répondants, admettent de nombreux problèmes de santé : traumatismes, douleurs articulaires (les genoux sont particulièrement sollicités) ; 61 d'entre eux disent avoir été opérés (ou sont sur le point de l'être) à la suite de traumatismes articulaires ;

- une fatigue partagée : presque un tiers des répondants affirme dormir en rentrant de cours épuisants (fréquemment ou tout le temps). 


\subsection{Les difficultés liées aux élèves}

32

\section{Concernant les difficultés mentionnées, les résultats les plus marquants sont les suivants : \\ - l'attitude des élèves semble pour eux un facteur de fatigabilité important. Ils désignent prioritairement leurs comportements déviants (118 désignations), leur inappétence pour l'effort (115), leur manque de goût pour la discipline (127), leur inattention (89). La facilité qu'ils ont d'échapper facilement au cours d'EPS (dispenses médicales par exemple) est désignée par une grosse moitié des enseignants ; \\ - Par ailleurs, deux tiers d'entre eux expriment trouver trop élevé le nombre d'élèves par classe. \\ 4.3 Les difficultés liées au collectif et à l'organisation de l'établissement}

Des items relatifs au collectif d'enseignants et à l'organisation du travail apparaissent également :

- le travail collectif au sein des équipes de professeurs d'EPS d'un établissement apparaitt comme une difficulté du métier pour une part non négligeable d'entre eux; 32 sur 218 réfutent ainsi l'idée d'une équipe soudée au sein de chaque établissement ; 42 pensent que cela se dégrade au fil du temps et 55 en font un facteur d'épuisement. Les femmes, notamment les plus de 40 ans, disent plus que les hommes ressentir la difficulté de vivre le collectif enseignant ${ }^{3}$;

- la mise en jeu de la responsabilité pénale de l'enseignant en cas d'accident d'élève semble largement redoutée par 170 des répondants, le même nombre d'entre eux pensent que cette mise en jeu possible s'aggrave d'année en année, et 160 en font une cause d'épuisement professionnel ${ }^{4}$;

- 38 enseignants jugent «intolérables» les charges de travail, et cet effectif monte à 119 s'agissant des charges extérieures à l'enseignement (administration, diverses réunions, bulletins, etc.) ;

- les plaintes concernant les conditions environnementales de l'exercice professionnel (froid, pluie, bruit...) sont mentionnées par une bonne moitié des répondants.

\subsection{Les difficultés relatives à l'institution et au statut social}

Par ailleurs, malaise et inquiétudes se décèlent dans le rapport à l'institution, à l'administration, et à la société :

- un quart des enseignants jugent «intolérable » le «poids des textes», ce qui renvoie aux prescriptions institutionnelles;

- le statut d'enseignant d'EPS et le niveau de reconnaissance sociale sont ressentis comme intolérables par respectivement 147 et 86 des répondants, et sont de plus désignés comme se délitant au fil du temps, pour le statut par 177 et pour la reconnaissance sociale par 78 enseignants;

- les enseignants ne sont pas globalement opposés à la bivalence ${ }^{5}$ : seulement un quart d'entre eux disent leur opposition au principe ;

- la «tenue des rôles que l'on est parfois amené à exercer à l'école » est considérée comme « intolérable » par 133 répondants ; 
- deux tiers des enseignants d'EPS interrogés accepteraient de prendre la retraite dès maintenant si elle leur était donnée à taux plein et presque la moitié d'entre eux envisageraient de changer d'emploi pour un salaire équivalent.

\subsection{Les hommes et les femmes se distinguent du point de vue des difficultés rencontrées}

Tableau 1. Sexe et difficultés du travail

\begin{tabular}{|c|c|c|c|}
\hline Cotation $>4$ & $\begin{array}{l}\text { \% } \\
\text { hommes }\end{array}$ & $\begin{array}{l}\% \\
\text { femmes }\end{array}$ & $\begin{array}{l}\text { p } \\
\text { (Chi-2) }\end{array}$ \\
\hline Facilité qu'ont les élèves à échapper au cours d'EPS : épuisant & 35,2 & 51,0 & $1,9 \%$ \\
\hline Charge de travail, temps passé au travail : intolérable & 21,3 & 13,6 & $13,6 \%$ \\
\hline Tenue de divers rôles : intolérable & 52,8 & 69,1 & $1,3 \%$ \\
\hline Tenue de divers rôles : va de + en + mal & 74,1 & 89,1 & $0,4 \%$ \\
\hline Tenue de divers rôles : épuisant & 78,7 & 89,1 & $3,7 \%$ \\
\hline $\begin{array}{l}\text { Conditions «environnementales» (chaleur, froid, bruit) : } \\
\text { intolérable }\end{array}$ & 50,9 & 61,8 & $10,5 \%$ \\
\hline Statut, promo, revenus, pouvoir d'achat : va de + en + mal & 88,0 & 74,5 & $1,1 \%$ \\
\hline Statut, promo, revenus, pouvoir d'achat : épuisant & 67,6 & 55,4 & $6,6 \%$ \\
\hline Collaboration des collègues : intolérable & 10,2 & 19,1 & $6,3 \%$ \\
\hline Collaboration des collègues : va de + en + mal & 13,9 & 24,5 & $4,6 \%$ \\
\hline Collaboration des collègues : épuisant & 20,4 & 30,0 & $10,2 \%$ \\
\hline $\begin{array}{l}\text { Rôle des parents d'élèves et importance qui leur est donnée } \\
\text { :箞 intolérable }\end{array}$ & 29,6 & 42,7 & $4,4 \%$ \\
\hline Éprouver une désillusion à propos du métier & 40,7 & 24,5 & $1,1 \%$ \\
\hline Éprouver une forme de lassitude par rapport au métier & 35,2 & 25,4 & $11,8 \%$ \\
\hline Peu de goût des élèves pour l'EPS (cotation $\leq 4$ ) & 36,1 & 47,3 & $9,5 \%$ \\
\hline
\end{tabular}

Le tableau 1 présente les pourcentages d'individus chez les hommes ( $1^{\text {re }}$ colonne) et les femmes ( $2^{\mathrm{e}}$ colonne) répondant être en accord avec les propositions figurant en ligne. La $3^{e}$ colonne indique la p-value issue du test du Chi- 2 d'interaction, exprimant la probabilité que les différences observées entre hommes et femmes soient uniquement dues au hasard (plus le pourcentage est petit, plus on peut avoir de certitude qu'il existe bien une différence entre les hommes et les femmes enquêtés). Ce tableau présente uniquement les 
variables du questionnaire pour lesquelles cette " part estimée de hasard » est inférieure ou égale à $15 \%$.

Les femmes semblent plus nombreuses (en proportion) que les hommes à être affectées par ce qui relève de problèmes pratiques et d'organisation, alors que c'est l'inverse pour les items qui touchent au ressenti et à la reconnaissance associés au métier. En effet, les hommes semblent éprouver plus de lassitude et de désillusion dans l'exercice de leur métier et sont notamment plus sensibles à la dégradation de la reconnaissance professionnelle; les femmes sont quant à elles plus sensibles aux problèmes de cœur de métier : les conditions de collaboration, le désengagement des élèves, le poids des parents à l'école qu'elles jugent excessif, la nécessité d'endosser de concert plusieurs rôles (éducatrice, surveillante, enseignante...).

Tableau 2. Sexe et problèmes de santé

\begin{tabular}{|l|l|l|l|}
\hline & $\begin{array}{l}\% \\
\text { hommes }\end{array}$ & $\begin{array}{l}\% \\
\text { femmes }\end{array}$ & $\begin{array}{l}\text { p } \\
\text { (Chi-2) }\end{array}$ \\
\hline $\begin{array}{l}\text { Éprouver des problèmes physiques/psychiques dus à la } \\
\text { pratique sportive passée }\end{array}$ & 49,1 & 39,1 & $13,8 \%$ \\
\hline $\begin{array}{l}\text { S'être rendu chez le médecin de famille dans les 6 derniers } \\
\text { mois pour cause de fatigue }\end{array}$ & 20,0 & 37,6 & $0,4 \%$ \\
\hline Avoir eu un arrêt de travail au cours des 6 derniers mois & 17,8 & 26,7 & $11,8 \%$ \\
\hline
\end{tabular}

Le tableau 2 présente les items de santé pour lesquels on trouve une différence $(p \leq 0,15)$ entre hommes et femmes. On observe que les femmes ont globalement plus de problèmes de santé que les hommes, mais qu'en revanche ceux-ci en signalent davantage qui soient en lien avec la pratique sportive passée.

\subsection{Les plus âgés font davantage état de problèmes tout en appréciant plus positivement leur travail}

Tableau 3. Âge et difficultés du travail

\begin{tabular}{|l|l|l|l|}
\hline Cotation > 4 & $\begin{array}{l}\%<42 \\
\text { ans }\end{array}$ & $\begin{array}{l}\text { \% } \\
\text { ans }\end{array}$ & $\begin{array}{l}\mathbf{p} \\
\text { (Chi-2) }\end{array}$ \\
\hline $\begin{array}{l}\text { Possibilité de travailler avec sa classe seul sur un lieu : va de + } \\
\text { en + mal }\end{array}$ & 22,9 & 15,2 & $14,9 \%$ \\
\hline Poids des textes officiels : va de + en + mal & 24,8 & 34,8 & $10,6 \%$ \\
\hline Charges extérieures à l'enseignement : épuisant & 62,9 & 75,0 & $5,3 \%$ \\
\hline $\begin{array}{l}\text { Rôle des parents d'élèves et importance qui leur est donnée : } \\
\text { intolérable }\end{array}$ & 31,4 & 41,1 & $14,0 \%$ \\
\hline
\end{tabular}




\begin{tabular}{|l|l|l|l|}
\hline $\begin{array}{l}\text { Rôle des parents d'élèves et importance qui leur est donnée : } \\
\text { va de + en + mal }\end{array}$ & 40,9 & 51,8 & $11,0 \%$ \\
\hline $\begin{array}{l}\text { Rôle des parents d'élèves et importance qui leur est donnée : } \\
\text { épuisant }\end{array}$ & 32,4 & 46,4 & $3,4 \%$ \\
\hline Utilité sociale comme prof d'EPS & 69,5 & 82,1 & $2,9 \%$ \\
\hline Efficacité dans l'exercice du métier & 66,7 & 81,2 & $1,4 \%$ \\
\hline Éprouver une forme de lassitude à propos du métier & 25,7 & 34,8 & $14,5 \%$ \\
\hline Éprouver une forme de stress à propos du métier & 29,5 & 44,6 & $2,1 \%$ \\
\hline Inattention des élèves (cotation <5) & 35,2 & 46,4 & $9,4 \%$ \\
\hline
\end{tabular}

Afin d'effectuer des comparaisons par âge, la population des enquêtés a été scindée en deux parties égales (42 ans est l'âge médian dans la population des répondants : 105 individus de moins de 42 ans, 112 de 42 ans et plus). Notons que dans le tableau 3, les pourcentages en vert sont associés à des appréciations "positives" sur le travail, contrairement à ceux en violet qui sont associés à des appréciations « négatives ».

On retiendra ici que :

- pour la plupart des items, les plus âgés sont plus nombreux en proportion à faire part de différentes difficultés liées à la pratique de leur métier ;

- en revanche, ils s'y estiment plus souvent efficaces ou socialement utiles que leurs cadets (auto-appréciation plus positive).

Figure 1. "Au cours des 5 dernières années, ce que l'on appelle globalement votre « forme » s'estelle dégradée?»

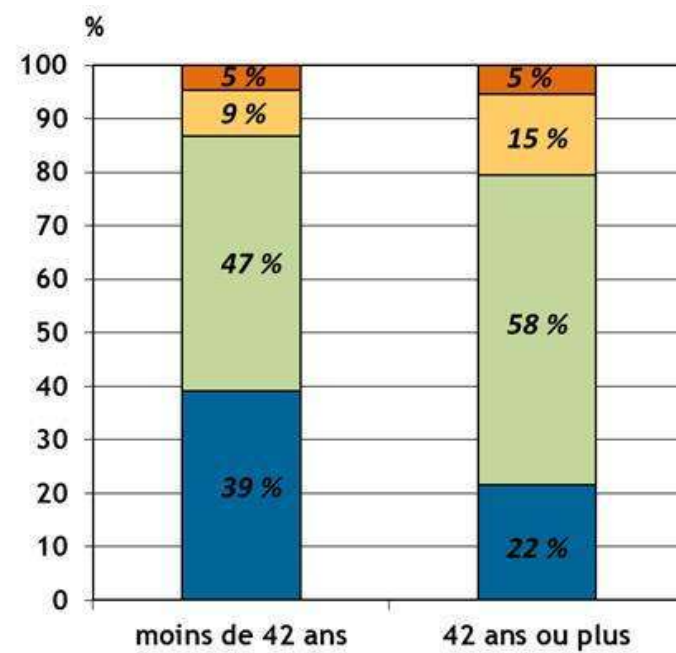

$\square$ J'ai de gros problèmes de santé

口J'ai souvent des problèmes de santé

$\square$ J'admets de temps à autres quelques problèmes de santé

$\square$ Pas du tout, je suis toujours en pleine forme

test du Chi-2: $p=3,3 \%$

La figure 1 montre que les gros problèmes de santé semblent concerner aussi peu les deux classes d'âge, sans doute parce que nombre des enseignants d'EPS atteints de pathologies lourdes sont amenés à quitter le métier. Les plus âgés sont en revanche plus nombreux en proportion que leurs cadets à déclarer avoir souvent des problèmes de santé ou à admettre de temps en temps quelques problèmes de santé, au détriment de la réponse « je 
suis toujours en pleine forme ", sachant que la probabilité que les différences observées soient dues au hasard n'est que de 3,3\%.

Tableau 4. Âge et santé

\begin{tabular}{|l|l|l|l|}
\hline & $\begin{array}{l}\mathbf{\%}<\mathbf{4 2} \geq \mathbf{4 2} \\
\text { ans }\end{array}$ & $\begin{array}{l}\mathbf{p} \\
\text { ans }\end{array}$ & (Chi-2) \\
\hline Éprouver des problèmes physiques/psychiques & 88,6 & 94,6 & $10,5 \%$ \\
\hline $\begin{array}{l}\text { Éprouver des problèmes physiques/psychiques dus à la } \\
\text { pratique professionnelle }\end{array}$ & 30,5 & 46,4 & $1,6 \%$ \\
\hline $\begin{array}{l}\text { Éprouver des problèmes physiques/psychiques dus à des } \\
\text { phénomènes liés à l'âge }\end{array}$ & 40,9 & 66,1 & $<0,1 \%$ \\
\hline Condition physique actuelle «mauvaise, je suis usé » & 2,9 & 8,9 & $6,0 \%$ \\
\hline Condition physique actuelle «moyenne, je me sens fatigué » & 18,1 & 27,7 & $9,4 \%$ \\
\hline Problème de santé récemment & 43,3 & 56,1 & $6,3 \%$ \\
\hline Suivre un traitement pour des problèmes ostéo-articulaires & 17,5 & 26,1 & $12,7 \%$ \\
\hline
\end{tabular}

41 Le tableau 4 présente les items de santé pour lesquels on trouve une différence $(p \leq 0.15)$ par âge. On observe que les plus âgés sont ceux qui déclarent le plus souvent éprouver un " problème de santé ».

Dans ce qui suit, des précisions seront apportées en croisant ces différents items par une technique d'analyse factorielle (Escofier et Pagès, 2008) permettant d'aboutir à une typologie des modalités de réponses au questionnaire.

\subsection{L'association des difficultés à des problèmes d'organisation du travail, de charge de travail et de sens du travail}

Une analyse des correspondances multiples (ACM) a été réalisée, pour étudier graphiquement la population des répondants, décrits par les variables qualitatives que constituent leurs réponses au questionnaire. Les variables dont les modalités vont servir à la construction des axes sont appelées variables actives. Il s'agit ici de 50 questions sur le travail, notamment celles dont les réponses se faisaient en cotant de 1 à 7 les axes de la tolérance («Tout à fait recevable » à « Intolérable »), diachronique («Cela va mieux qu'il y a quelques années » à «Cela va de plus en plus mal ») et de fatigabilité («Aucunement fatigant » à «Épuisant »). Des variables supplémentaires peuvent figurer sur le graphique sans avoir servi à la construction des axes. Il s'agira ici notamment des questions sur la santé.

Rappelons que l'interprétation d'une telle analyse se fait ainsi: deux modalités de réponse "se ressemblent » d'autant plus lorsque les individus qui ont répondu l'une ont souvent aussi répondu l'autre. Graphiquement, cela se traduit par la proximité dans l'espace des deux points qui représentent ces deux modalités. Au contraire, plus deux 
points sont éloignés, plus les individus qui ont répondu l'une des deux modalités n'ont en général pas répondu l'autre. On obtient ainsi une représentation visuelle des corrélations entre les réponses données par les enseignants d'EPS au questionnaire. Par ailleurs, plus un point est éloigné de l'origine des axes, moins la modalité de réponse associée a été fréquemment donnée par les individus enquêtés.

Figure 2. « Paysage travail » sur l'axe de la tolérabilité

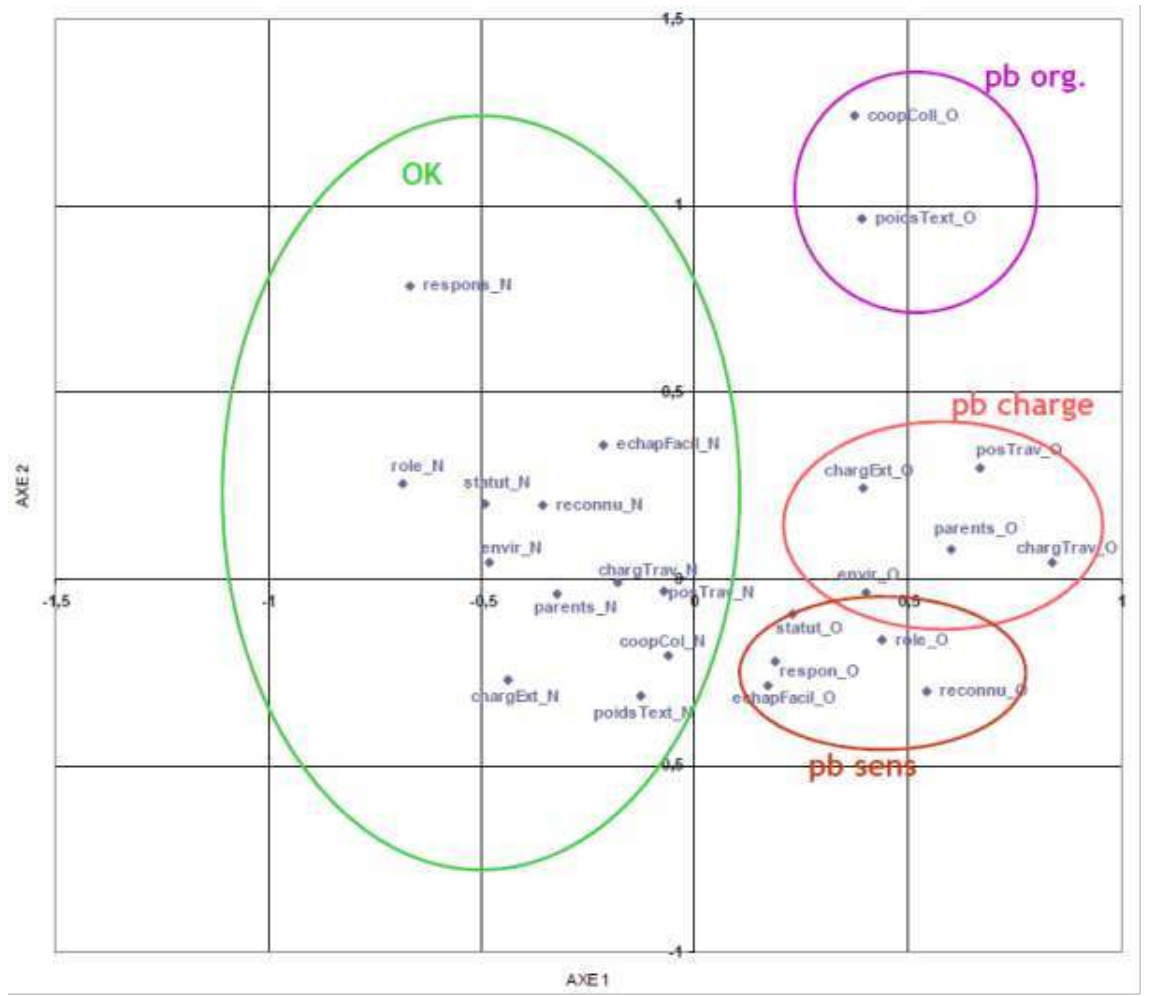

La figure 2 (voir annexe pour la signification des variables représentées), sur laquelle sont représentées les réponses sur l'axe de la tolérance, permet de distinguer quatre zones de réponses. On voit que tout l'ouest du graphique correspond aux réponses "positives " (zone « $\mathrm{OK}$ », en vert), et l'est aux réponses «négatives». Parmi ces dernières, on distingue au nord-est une zone correspondant aux problèmes d'organisation du travail (" pb org. ", en rose), à l'est une zone regroupant des réponses concernant des difficultés liées à la charge de travail (" pb charge », en rouge), et un peu plus au sud les réponses faisant état de problèmes renvoyant au sens du travail («pb sens », en marron).

On retrouve la même organisation des modalités de réponses pour les deux autres axes de cotation (diachronique et de la fatigabilité): les zones décrites fonctionnent pour l'ensemble des variables actives introduites dans l'analyse.

La figure 3 présente les croisements de quelques variables deux à deux, pour chaque zone de réponses négatives, pour vérifier les corrélations mises en évidence par l'ACM.

Pour la zone relevant des problèmes plutôt organisationnels, on voit par exemple que les individus qui trouvent intolérable le manque de collaboration entre collègues sont plus nombreux (en proportion) que les autres à trouver intolérable le poids des textes (prescriptions institutionnelles portant sur le travail) : 40,6\% contre 23,7\% (tableau du haut). Autrement dit, le poids des textes est plus souvent vécu comme intolérable par les individus ayant un problème du côté de la coopération que par ceux qui n'en ont pas. 
Pour la zone ayant trait aux problèmes liés à la charge de travail, on fait de même en croisant la possibilité de travailler avec sa classe seul sur un lieu (selon l'axe de fatigabilité) et la charge de travail (selon l'axe de la tolérance) d'une part ( $2^{\mathrm{e}}$ tableau), et ces deux mêmes variables sur l'axe diachronique d'autre part ( $3^{e}$ tableau). Dans les deux cas, la charge de travail est bien corrélée au manque de possibilité de travailler avec la classe.

Pour la zone référant les difficultés liées au sens du travail, on croise la reconnaissance sociale du professeur d'EPS, selon l'axe de la tolérance, avec son point de vue sur l'attitude des élèves, une attitude inacceptable étant liée au manque de reconnaissance sociale ressenti ( $4^{\mathrm{e}}$ tableau). On croise aussi la reconnaissance sociale du professeur d'EPS, mais cette fois selon l'axe diachronique, avec la facilité qu'ont les élèves à échapper au cours d'EPS sur ce même axe (5 $5^{e}$ tableau). La dégradation de l'un dans le temps est bien liée, fortement, à la dégradation de l'autre.

Figure 3. Vérification de corrélations mises en évidence par l'ACM

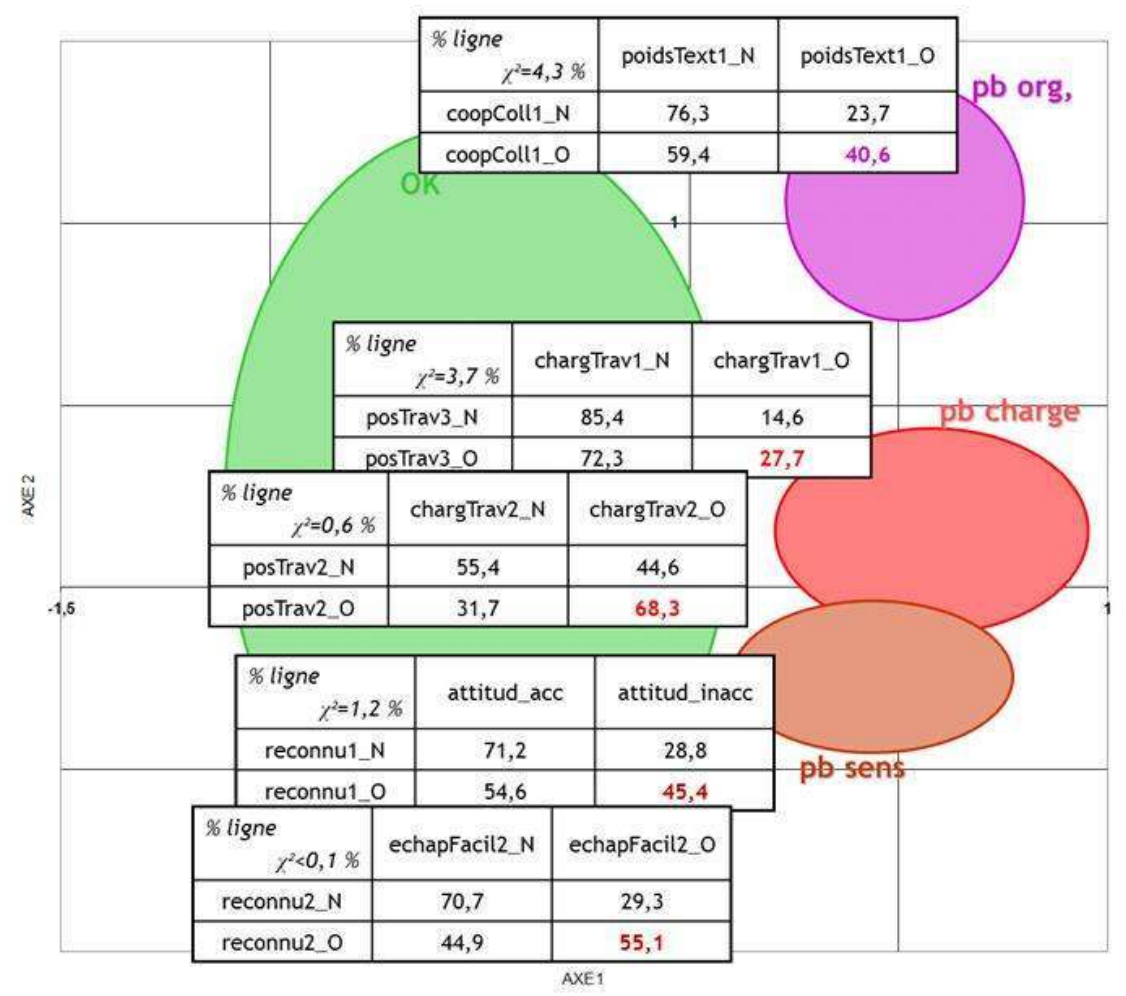

\subsection{Les relations santé-travail : certains aspects de la santé des enseignants enquêtés semblent liés au travail de façon différenciée}

En projetant la santé en variables supplémentaires sur ce "paysage travail » (figure 4), nous nous sommes intéressés plus particulièrement à trois aspects : la dégradation de la «forme » ces cinq dernières années (avec des modalités de réponse allant de «je suis en pleine forme » à «j'ai de gros problèmes de santé ») ; se sentir épuisé, voire dormir en rentrant d'une journée chargée en cours (réponses de « jamais » à « toujours »); enfin, les troubles du sommeil causés par les problèmes professionnels (de "jamais » à «toujours »). Nous constatons que, pour ces trois questions, les modalités de réponse 
forment à chaque fois un axe allant de la zone « $\mathrm{OK}$ » vers les zones représentant des problèmes dans le travail, en différenciant cependant " sommeil troublé toujours ", qui se retrouve du côté des problèmes d'organisation, et «épuisement toujours » du côté des problèmes de sens du travail. Les " gros problèmes de santé " se situent entre les deux au niveau de la zone représentant les problèmes de charge.

Figure 4. Éléments de santé projetés sur le « paysage travail »

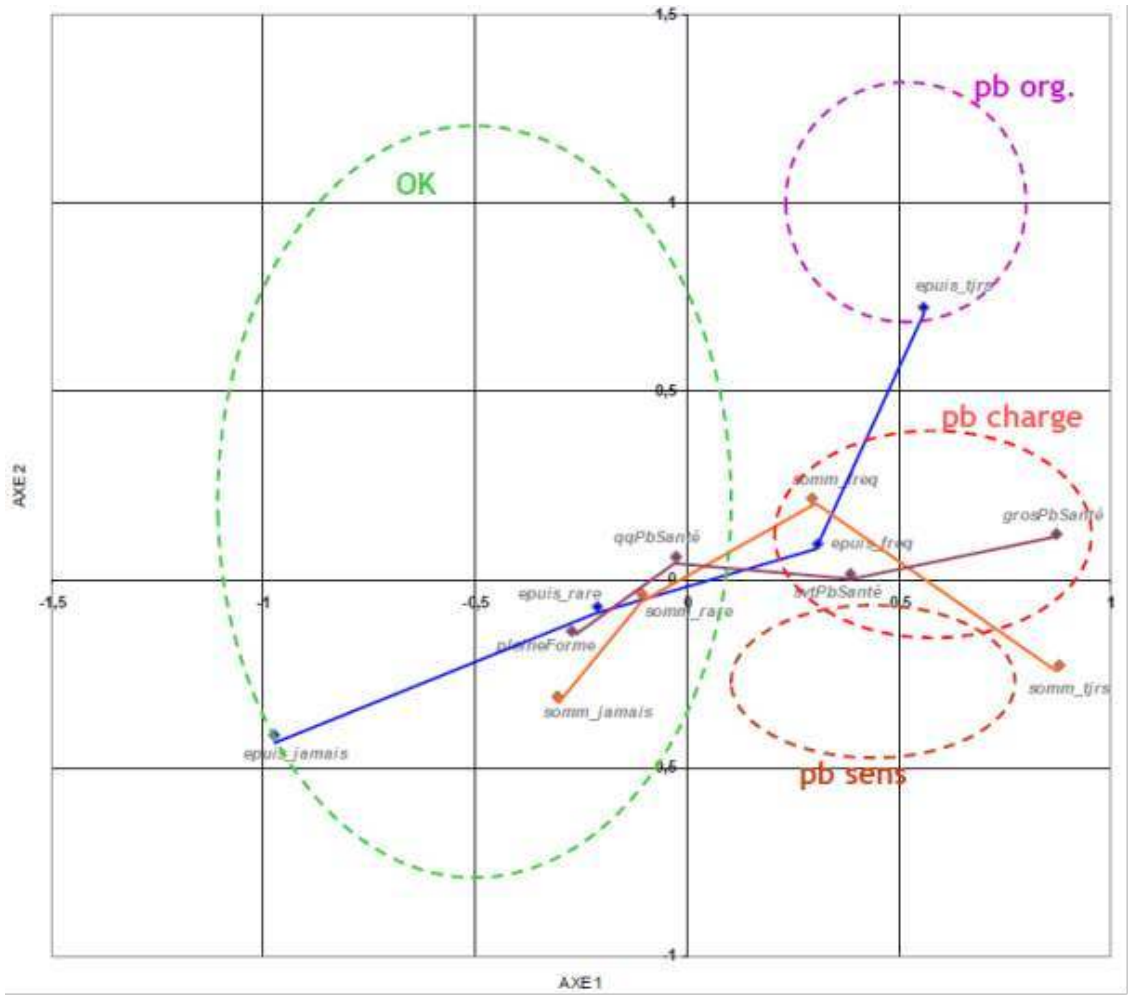

\section{entre ces problèmes de santé et les différents aspects négatifs du travail dégagés dans} l'ACM.

Tout d'abord, une question a été choisie comme proxy de chaque zone de problèmes liés au travail :

- la zone ayant trait aux problèmes d'ordre organisationnel ( $\mathrm{pb}$ org) est représentée ci-après par la question sur « La collaboration des collègues de l'équipe EPS de votre établissement », de « pas fatigant » à « épuisant »;

- la zone de problèmes liés à la charge ( $\mathrm{pb}$ charge) par la question sur «Les charges extérieures à l'enseignement (administration, les diverses réunions, les bulletins...)» (également selon le caractère « fatigant »);

- la zone regroupant plutôt les problèmes liés au sens du travail ( $\mathrm{pb}$ sens) par la question sur «La facilité qu'ont les élèves à échapper au cours d'EPS (mot des parents, complaisance à propos de « douleurs » passagères...) » (caractère « fatigant »).

Chacune de ces variables travail a été croisée avec la question santé qui semble lui correspondre au vu de l'ACM (par exemple, le proxy des problèmes organisationnels avec la question sur l'épuisement), mais aussi avec les questions santé en rapport avec les autres zones de problèmes liés au travail. 
Tableau 5. p-value pour les croisements entre les variables de travail en ligne et les variables de santé en colonne

\begin{tabular}{|l|l|l|l|}
\hline p-value (Chi-2 d'interaction) & Épuisement & « Forme » & Troubles du sommeil \\
\hline $\begin{array}{l}\text { Pb coop avec les collègues } \\
\text { (zone pb orga) }\end{array}$ & 0,07 & 0,56 & $<0,01$ \\
\hline $\begin{array}{l}\text { Charge extérieure aux enseignements } \\
\text { (zone pb charge) }\end{array}$ & 0,03 & 0,02 & 0,09 \\
\hline $\begin{array}{l}\text { Elèves qui échappent aux cours d'EPS } \\
\text { (zone pb sens) }\end{array}$ & 0,09 & 0,35 & 0,02 \\
\hline
\end{tabular}

Le tableau 5 présente la valeur des seuils issus des tests de Chi-2 d'interaction pour chacun de ces croisements. Il s'agit d'une indication sur la significativité du lien entre deux variables que l'on croise : on l'a dit, plus le seuil est petit, plus le lien est significatif. Chaque case colorée indique un croisement entre un proxy travail et un aspect de la santé repéré comme lui étant lié dans l'ACM ; comme attendu, le lien entre une variable travail caractérisant une zone de l'ACM et la variable santé dont la " pire » modalité se retrouve dans la même zone est tout à fait significatif.

La variable sur la « forme » ne semble ici liée qu'à la charge extérieure aux enseignements $(p=0.02$, alors que pour les problèmes de coopération et la facilité qu'ont les élèves à échapper aux cours d'EPS, $p$ est respectivement égal à 0,56 et 0,35 ). En revanche, les autres variables de santé sont liées à toutes les variables travail (et donc à chaque zone définie à partir de l'ACM), puisque p est dans tous les cas inférieur à $10 \%$. Le fait d'avoir considéré ces variables travail selon leur caractère plus ou moins « fatigant » joue très certainement un rôle dans ce résultat.

Ce dernier constat nous a amenés à procéder à des régressions logistiques, avec comme variables à expliquer successivement les trois variables de santé, et comme variables explicatives le sexe, l'âge, et les trois variables travail choisies comme proxy des zones identifiées avec l'ACM. Pour chaque variable de santé à expliquer, ceci a été fait trois fois, avec chacune des trois échelles de cotation proposées pour les variables travail ; ce sont en tout neuf régressions qui ont été ainsi mises en œuvre. Les principaux résultats en étaient les suivants (odds-ratios significativement différents de 1 avec une marge d'erreur de $1 \%(* *)$ ou $5 \%(*)$, valeur précisée entre parenthèses) :

- Effets sur la santé pris sous l'angle "aucunement fatigant " à "épuisant» (axe dit de fatigabilité) :

- Toutes choses égales par ailleurs, la charge extérieure à l'enseignement jugée épuisante est ce qui augmente le plus la probabilité de se sentir épuisé en rentrant d'une journée chargée $\left(\mathrm{OR}=1,8^{*}\right)$, et aussi la probabilité d'avoir une forme dégradée ces cinq dernières années $\left(\mathrm{OR}=2,35^{*}\right)$.

- La probabilité des troubles du sommeil augmente avec le fait de trouver épuisant que les élèves échappent facilement aux cours $\left(\mathrm{OR}=1,92^{*}\right)$, et surtout avec les problèmes de coopération vus comme épuisants ( $\left.\mathrm{OR}=2,41^{* *}\right)$. 
- Effets sur la santé pris sous l'angle de l'évolution ces cinq dernières années (axe dit diachronique) :

- La probabilité de se sentir épuisé en rentrant d'une journée chargée augmente avec la dégradation sur les cinq dernières années de la charge $\left(\mathrm{OR}=1,90^{*}\right)$ et plus encore de la coopération $(\mathrm{OR}=2,50 *)$.

- La dégradation de la charge est ce qui augmente significativement la probabilité d'occurrence des troubles du sommeil ( $\left.\mathrm{OR}=2,52^{* *}\right)$.

- Effets sur la santé pris sous l'angle «tout à fait recevable» à «intolérable » (axe dit de tolérance) :

- Un peu comme précédemment, la probabilité de se sentir épuisé en rentrant d'une journée chargée augmente avec le fait de trouver intolérable la charge $\left(\mathrm{OR}=2,36^{*}\right)$ et les problèmes de coopération $\left(\mathrm{OR}=2,33^{*}\right)$. et coll, 2007). On l'a dit, deux chercheurs en sciences et techniques des activités physiques et sportives et en sciences de l'éducation sont à l'initiative de l'enquête, leur souci d'experts formateurs étant d'objectiver leur perception de difficultés croissantes pour les enseignants d'EPS à vivre et à vieillir dans leur travail. De ce point de vue-là, l'objectif initial paraît rempli.

61 Tardivement impliqués dans la construction de l'enquête, les ergonomes ont ici essentiellement piloté l'interprétation et la discussion des résultats. Il faut donc noter qu'à l'origine de cette étude, il n'y a pas de demande (au sens ergonomique), pas de demandeur du milieu professionnel considéré. On peut considérer cette étude comme un levier de prise de conscience par la communauté professionnelle concernée des difficultés réelles existantes d'une part, et des possibilités de changement d'autre part. On peut considérer que l'approche ergonomique est essentielle de ce point de vue-là : dépasser la simple description des difficultés pour favoriser la perception de changements possibles. On peut donc espérer que cette étude contribue à faire émerger une demande sociale propice au développement des méthodologies ergonomiques d'intervention plus classiques, qui incluraient à la fois des analyses qualitatives et contextualisées de situations de travail et un accompagnement dans la recherche de solutions. La nature des résultats qui sont repris ci-dessous renforce la pertinence de l'approche ergonomique pour comprendre et transformer des situations de travail des enseignants d'EPS et dans l'enseignement général plus globalement. 


\subsection{Les limites de l'étude et les développements potentiels ultérieurs, notamment autour des questions de genre} résultats nous amènent à considérer que le repérage et l'investigation des différentes facettes de la fatigue des enseignants d'EPS (selon la source de la fatigue, la façon dont elle se traduit, etc.) et de leurs liens avec leur travail constitueraient une orientation intéressante pour de futurs travaux de terrain. En d'autres termes, il s'agirait de repérer si certains aspects du travail sont liés avec certaines "manières d'être fatigué ", si certaines modalités de fatigue sont davantage associées à certaines difficultés du travail, 
ou inversement, si l'absence de ces symptômes est liée à la présence de certaines conditions ou ressources. À nouveau, une attention particulière pourrait être portée aux distinctions relatives au sexe.

\subsection{Les perspectives de transformation du point de vue de l'ergonomie}

67 Si les résultats présentés dans cette enquête méritaient d'être précisés sur certains aspects, des conséquences concrètes peuvent malgré tout être tirées dès à présent du point de vue des perspectives d'action envisageables.

\subsubsection{La gestion des fins de carrière}

Les résultats de cette enquête, comme ceux de travaux antérieurs pour d'autres enseignants (Cau-Bareille, 2009), soulignent les difficultés du vieillissement au travail pour les enseignants d'EPS, bien présents dans le tableau 4 : la diminution des capacités physiques qui altère les possibilités de démonstration, de parades, et donc la crédibilité accordée par les élèves notamment; la fatigue accumulée et les blessures associées à une population sportive de travailleurs, etc. Tout cela contribue à rendre compliquées les fins de carrière pour de nombreux enseignants d'EPS. Des aménagements permettraient certainement de préserver leur engagement: si les plus âgés manifestent plus de difficultés, ils sont aussi ceux qui mettent le plus en avant un sentiment d'utilité et d'efficacité. Le fait qu'ils manifestent également le plus de désillusion et de stress peut être interprété comme une perception, accrue du fait de leur ancienneté, de la dégradation des conditions du métier. L'ouverture de ces enseignants à la bivalence (seuls $24,8 \%$ s'y opposent dans notre étude) peut être interprétée comme témoignant d'un besoin de changement, même si la corrélation à l'âge n'est pas démontrée.

Ces aménagements de fin de carrière dépendent en partie des possibilités institutionnelles et statutaires. Certains dépendent probablement aussi des marges de manœuvre qu'offrent les collectifs de travail et les administrations des établissements. De ce point de vue-là, des études ergonomiques de terrain seraient certainement précieuses pour identifier les régulations possibles.

\subsubsection{Le travail collectif, l'organisation et ses empêchements}

70 Il est intéressant de noter que le discours général porté sur le travail est spontanément positif. Les enseignants mettent en avant leur vocation pour le métier, ou encore leur sentiment d'utilité. Mais les questions plus précises sur le travail apportent des réponses très différentes.

71 Ce décalage est relativement classique dans le monde du travail : il est plus précisément caractéristique des situations dites "d'amputation du pouvoir d'agir» (Clot, 2008; Coutarel, 2008;, 2013). Dejours (1993) a décrit ces mécanismes en termes de stratégies défensives de métier. On peut donc tout à fait concevoir que les réponses spontanées à caractère général des enseignants d'EPS relèvent de tels processus :

- elles paraissent confirmer le discours communément admis qui consiste à idéaliser ce métier et donc à rendre socialement illégitime la question des conditions de travail. Ceci conforte le poids du discours dominant sur les pratiques effectives et la manière d'en rendre compte ; 
- ensuite, elles peuvent traduire une forme de fatalisme : "Ça fait partie du métier ». Cette croyance pourrait être un refuge pour mieux vivre avec les difficultés quotidiennes, de la même manière qu'il serait «normal » pour un carreleur d'avoir mal aux genoux. Ce refuge est aussi une impasse, puisqu'il interdit toute forme d'action destinée à améliorer les situations concernées.

72 Ce décalage confirme aussi la nécessité de mobiliser des méthodes d'investigations spécifiques pour dépasser ces préjugés dont les acteurs concernés sont eux-mêmes porteurs. La fabrication d'autres possibles par l'intervention ergonomique (Coutarel et Daniellou, 2011) constitue certainement une voie pour sortir de ces impasses.

73 Nous avons été surpris de constater que le travail collectif, plutôt que de constituer majoritairement une ressource (Clot, 2008), était essentiellement perçu comme une difficulté. On peut penser que ce sont les conditions de mise en œuvre de ce travail collectif qui sont ici pointées du doigt. Plusieurs hypothèses convergentes peuvent expliquer ce résultat :

- le travail collectif impose le regard d'autrui sur sa pratique et donc ses faiblesses supposées, ses écarts consentis, dans un contexte où il apparaît difficile de tenir. La corrélation établie entre l'intolérabilité du poids des textes et les problèmes de coopération supporte cette interprétation;

- la difficulté à travailler en équipe n'est pas sans lien avec des éléments de genre que nous avons évoqués plus haut et qu'il faudrait prendre en compte ;

- le déficit de management intermédiaire pourrait faire l'objet d'une autre hypothèse en comparant la vie d'un établissement scolaire et la vie de l'entreprise. Il y a des limites importantes et évidentes à la comparaison entre les deux milieux professionnels, mais du point de vue de l'ergonomie de l'activité, le rôle de l'encadrement intermédiaire est bien d'organiser le travail, de construire les réponses aux difficultés du quotidien, de supporter les régulations pour en limiter le coup... dans un établissement scolaire, entre les enseignants d'EPS et le chef d'établissement, il n'y a personne pour jouer ce rôle. Le coordinateur EPS joue un rôle très technique qui ne recouvre pas ces fonctions d'organisation du travail ;

- les difficultés du travail favorisent le repli sur soi. Se couper des relations avec les autres collègues peut constituer une manière de se préserver de contraintes que l'on ne veut pas subir ;

- enfin, les difficultés de coopération dépassent le périmètre des enseignants d'EPS. La vie scolaire est largement co-construite par les interactions avec l'ensemble des professionnels qui agissent au sein de l'établissement. Les enseignants expriment souvent les difficultés vécues avec l'institution, l'administration. Du point de vue de l'ergonomie, la qualité des apprentissages des élèves ne peut être envisagée qu'à la seule lumière du travail enseignant : l'ensemble des professionnels de l'établissement (voire au-delà) contribuent d'une manière ou d'une autre, parfois moins visible, à la vie scolaire. Agents de service, personnel administratif, agents techniques, etc. revendiquent une contribution à l'éducation qui est très peu mise en avant. Dans un vocabulaire ergonomique, nous dirions que la coopération et la co-activité interpellent l'ensemble des acteurs du système établissement qui participent de différentes manières et à divers degrés de la production du service éducatif.

\subsubsection{L'intervention ergonomique en milieu scolaire}

74 Tous les éléments de discussion évoqués ici renforcent la pertinence d'envisager l'ergonomie comme une ressource potentielle dans la conception des situations de travail 
des enseignants d'EPS, et certainement des enseignants de manière plus générale. Les problèmes d'organisation, de charge de travail ou encore de sens du travail mis en avant par nos résultats constituent des problématiques habituelles pour l'ergonomie. Des expérimentations organisationnelles (Petit et Coutarel, 2013) à partir d'une analyse de l'activité des enseignants, mais aussi de la prise en compte des autres acteurs d'un établissement, pourraient être mises en œuvre dans le cadre d'une intervention ergonomique.

Tout ce qui touche à l'organisation de la vie de l'établissement relève de la responsabilité du chef d'établissement. En concertation avec le rectorat, le département ou la région, nous pouvons imaginer que des études pilotes permettraient d'établir des méthodologies d'intervention ergonomique adaptées dans un établissement où les différents acteurs seraient partie prenante.

\subsubsection{L'intervention ergonomique en conception}

\section{lycées. Depuis, c'est essentiellement dans d'autres domaines (conception d'usines, de} maisons de retraite, d'hôpitaux, de services d'assurance ou de banques...) que l'ergonomie a pu investir les processus de conception et affirmer sa pertinence et ses méthodologies d'intervention en conception (Daniellou, 2004; Martin, 2004). Si les occasions d'intégrer très tôt ces processus de conception dans le système éducatif sont peu nombreuses, il reste évident que les apports d'une analyse ergonomique du travail qui fournisse des repères de conception fondés sur une connaissance du travail et des usages réels permettraient de les optimiser significativement.

\subsubsection{La formation continue des enseignants et l'ergonomie}

79 La formation des adultes est une préoccupation forte de l'ergonomie de l'activité depuis de nombreuses années. La formation à partir de l'analyse ergonomique du travail ou la formation à l'analyse du travail constituent par exemple des leviers d'intervention importants pour le développement des professionnels concernés.

80 Si agir en faveur de la santé des enseignants semble devoir passer par la construction de nouvelles ressources et possibilités pour les enseignants d'influencer les conditions de leur travail, il serait certainement intéressant d'évaluer l'apport de dispositifs de formation continue mobilisant l'ergonomie, dans la lignée des travaux ergonomiques relatifs à la formation et au développement des compétences (Dugué et coll., 2010 ; Delgoulet et Vidal-Gomel, 2013; Six-Touchard et Falzon, 2013). La formation-action est ainsi un mode d'intervention ergonomique de plus en plus mobilisé aujourd'hui. 
81 La formation continue est totalement intégrée au processus de gestion de la carrière des enseignants par le système éducatif. Les enseignants d'EPS peuvent tous les ans demander et suivre des stages proposés par le rectorat. Pour le moment, ces stages ont essentiellement pour objet des compétences techniques relatives aux savoirs disciplinaires, aux connaissances juridiques et réglementaires. Nos résultats conduisent cependant à interroger cette offre de formation continue qui semble peu répondre aux problèmes d'organisation, de charge de travail et de sens du travail, eux-mêmes associés à des symptômes concernant la santé.

On peut penser que les connaissances générales sur les relations santé-travail et sur les conditions aujourd'hui connues d'atteinte à la santé et/ou de développement des acteurs par le travail pourraient rendre les enseignants capables d'interroger le système dans lequel ils s'inscrivent à partir d'outils basiques d'analyse du travail et de conduite collective de projet. Des formations pilotes pourraient en ce sens être mises en place relativement facilement et évaluées.

De manière encore plus ambitieuse, le même type de formation adressé aux autres personnels des établissements pourrait permettre de faire évoluer les relations interprofessionnelles existantes vers des formes plus collaboratives et constructives. En effet, les difficultés des uns et des autres ont essentiellement pour effet le repli sur soi et l'opposition des collectifs d'acteurs dans des relations professionnelles crispées qui ne font que diminuer les ressources disponibles (ou tout du moins leur perception) pour chacun et multiplier les situations d'injonctions contradictoires.

Si les dimensions collectives et organisationnelles de l'activité de l'enseignant s'avèrent déterminantes quant aux possibilités d'un développement conjoint des apprentissages des élèves et de l'enseignant lui-même par le travail, l'approche ergonomique peut certainement constituer un levier pour la formation continue des personnels et le maintien d'un engagement favorable à la qualité des apprentissages des élèves.

\section{Conclusion}

Les résultats que nous présentons sont assez conformes à ceux de la littérature (par exemple Bluteau, 2008) et les précisent : au-delà d'une image d'eux-mêmes qu'ils veulent valorisante, d'une « vocation » qui perdure au fil du temps, d'une croyance farouche dans les bienfaits de leur discipline, les enseignants d'EPS ne sont pas exempts de difficultés professionnelles, elles-mêmes associées à des difficultés de santé.

Les experts s'accordent à dire que l'enseignant d'EPS, sans être un enseignant «à part » dans la communauté éducative, exerce un métier physiquement et psychologiquement éprouvant : des facteurs de risques d'atteintes à la santé au travail identifiés pour d'autres populations de travailleurs semblent présents dans ses situations professionnelles. Malgré cela, la question de sa santé au travail n'est qu'exceptionnellement évoquée. L'allongement $\mathrm{du}$ temps de présence au travail pose et posera nécessairement des problèmes qui renforceront l'idée qu'il s'agit d'un métier difficile à exercer, pour lequel des perspectives d'évolution de carrière, de gestion de fin de carrière, d'amélioration des conditions de travail sont sans doute à envisager. Nos résultats mettent en avant les problèmes d'organisation, de charge de travail et de sens du travail, significativement associés à des symptômes d'atteinte à la santé. Ces résultats confirment la pertinence d'une approche du travail enseignant centrée sur les conditions effectives de ce travail et 
le recours utile que peut représenter l'ergonomie. La conception, la formation continue, les projets de l'établissement constituent certainement des occasions propices pour évaluer l'apport de l'ergonomie aux problématiques soulevées.

Face aux difficultés professionnelles qui s'installent, la littérature en santé au travail a pu mettre en évidence deux types de réponse : une "surmobilisation" très coûteuse du professionnel qui "prend sur lui», jusqu'à l'atteinte à la santé, sur les dimensions physique (troubles musculo-squelettiques, lombalgies, fatigue chronique...) et/ou mentale (atteinte à l'estime de soi, stress, dépression, suicide...), dont les liens étroits sont bien connus; un désengagement vis-à-vis du travail qui protège partiellement (posture de retrait, non implication, démission, changement d'orientation de carrière). Face à cette alternative, la question centrale de l'apprentissage des élèves ne peut plus faire l'économie d'un examen sérieux des conditions faites au travail. La très grande majorité des travaux en sciences de l'éducation n'interpelle le travail enseignant qu'à l'occasion de questions d'apprentissage et d'éducation de l'élève. L'ergonomie propose une approche qui prend en charge le travail enseignant pour lui-même, avec ses difficultés et ses dilemmes, au sein d'une approche systémique qui n'ignore pas l'ensemble des acteurs et des déterminants organisationnels.

\section{BIBLIOGRAPHIE}

Askenazy, P., Cartron, D., de Conninck, F., Gollac, M. (2006). Organisation et intensité du travail. Octarès Editions, Toulouse.

Bluteau, S. (2008). Rapport au métier et perception des conditions de travail chez les enseignants d'EPS. Rapport de recherche, Faculté des sciences du sport, Université Paris 1 Panthéon Sorbonne, CNRS.

Carraud, F. (2006). Apprendre et enseigner en ZEP. Dans, Apprendre et enseigner en « milieux difficiles ", ed Centre Alain Savary, pp. 31-43, INRP, Paris.

Cau-Bareille, D. (2009). Vécu du travail et santé des enseignants en fin de carrière : une approche ergonomique. CEE-CREAPT, Rapport de recherche 56.

Clot, Y. (2008). Travail et pouvoir d'agir. PUF, Paris.

Coutarel, F. (2008). Effort. Dans, Corps et Couleurs, ed. G. Boëtsch, D. Chevé, P. Blanchard, Éditions CNRS, Paris.

Coutarel, F. (2011). Quelle reconnaissance du travail réel, des tensions et déséquilibres dans la quête de performance. Dans, Les nouvelles organisations du travail : entre souffrance et performance, eds P. Chaudat, R. Muller, pp. 73-96, L'Harmattan, Paris.

Coutarel, F., Daniellou, F. (2011). L'intervention ergonomique pour la prévention des troubles musculo-squelettiques : quels statuts pour l'expérience et la subjectivité des travailleurs? Travail et Apprentissages, 7, 62-80.

Coutarel, F., Petit, J. (2013). Prévention des TMS et développement du pouvoir d'agir. Dans, Ergonomie constructive, ed P. Falzon, pp. 175-190, PUF, Paris. 
Daniellou, F. (2004). L'ergonomie dans la conduite de projets de conception de systèmes de travail. Dans, Ergonomie, ed P. Falzon, pp. 359-374, PUF, Paris.

Daniellou F., Rabardel P. (2005). Activity-oriented approaches to ergonomics : some traditions and communities. Theoretical Issues in Ergonomics Science, 6, 5, 353-357.

Davezies, P. (1999). Évolution des organisations du travail et atteintes à la santé. Travailler, 3, 87-114.

Delgoulet, C., Vidal-Gomel, C. (2013). Le développement des compétences : une condition pour la construction de la santé et de la performance au travail. Dans, Ergonomie constructive, ed P. Falzon, pp. 19-32, PUF, Paris.

Delvolvé, N., Margot, A. (2001). Le travail de l'enseignant du point de vue de l'ergonomie. Psychologie et éducation, 44, 43-54.

Dejours, C. (1993). Travail, usure mentale. Essai de psychopathologie du travail, de la psychologie à la psychodynamique du travail. Bayard éditions, Paris.

Dugué, B., Petit, J., Daniellou, F. (2010). L'intervention ergonomique comme acte pédagogique. PISTES, 12, 3. http://pistes.revues.org/2767

Escofier, B., Pagès, J. (2008). Analyses factorielles simples et multiples; objectifs, méthodes et interprétation. Dunod, $318 \mathrm{p}$.

Falzon, P. (2004), Nature, objectifs et connaissances de l'ergonomie. Dans, Ergonomie, ed P. Falzon, pp. 17-35, PUF, Paris.

Falzon, P., Teiger, C. (2011). Ergonomie, formation et transformation du travail. Dans, Traité des Sciences et Techniques de la formation, ed P. Caspar, P. Carré. Dunod, pp. 143-159.

Fiard, J., Jourdan, D., Simar, C. (2008). Du terrain de l'enquête à celui de la formation. Concevoir autrement la place de l'éducation à la santé dans la formation des enseignants d'EPS. Actes du colloque Un nouveau cadre pour l'éducation à la santé et la prévention des conduites addictives en I.U.F.M. Quels enjeux, quels dispositifs? Toulouse, Editions Universitaires du Sud.

Gal-Petitfaux, N., Saury, J. (2002). L'agir professionnel en éducation physique et en sport dans une perspective d'anthropologie cognitive. Revue française de pédagogie, 138, 51-61.

Guérin, F., Laville, A., Daniellou, F., Duraffourg, J., Kerguelen, A. (1997). Comprendre le travail pour le transformer. ANACT, Lyon.

Houssaye, J. (2000). Le triangle pédagogique. Théorie et pratiques de l'éducation scolaire. Peter Lang, Berne.

Lacomblez, M., Bellemare, M., Chatigny, C., Delgoulet, C., Re, A., Trudel, L., Vasconcelos, R. (2007). Ergonomics analysis of work activity and training : Basic paradigm, evolutions and challenges. Dans, Meeting Diversity in Ergonomics, eds R. Pikaar, E. Koningsveld, P. Settels, pp. 129-142, Elsevier. Lancry-Hoestlandt, A. (2013). À propos d'ergonomie scolaire. Dans, Travail, Conception, Santé, ed A. Drouin, pp. 381-389, Octarès.

Lambert, G., Pascal, R. (1983). Ergonomie scolaire et éducative. Bibliographie annotée. Paris, UNESCO.

Martin, C. (2004). L'ergonome dans les projets architecturaux. Dans Ergonomie, ed P. Falzon, pp. 421-436, PUF, Paris.

Messing, K., Chatigny, C. (2004). Travail et genre. Dans, Ergonomie, ed P. Falzon, pp. 301-316, PUF, Paris. 
Messing, K., Seifert A.M. (1997). Reine de la salle de classe. Dans, Stratégies et résistance du travail des femmes, ed A. Soares, pp. 69-106, Harmattan, Montréal.

Montmollin, M. de (1974). L'analyse du travail préalable à la formation. Armand Colin.

Montmollin, M. de. (1993) Compétences, charge mentale, stress : peut-on parler de santé« cognitive » ? Actes du XXVIII ${ }^{\mathrm{e}}$ Congrès de la SELF, Genève, 22-24 septembre.

Lipp, A., Ria, L. (2012). La transmission des savoirs en formation professionnelle initale : Analyse de l'activité d'enseignants en lycées agricoles. @ctivités, 9, 2, 71-87.

Petit, J., Coutarel, F. (2013). L'intervention comme dynamique de développement conjoint des acteurs et de l'organisation. Dans, Ergonomie constructive, ed P. Falzon, pp. 133-146, PUF, Paris.

Prévost, J., Messing, K. (2001). Stratégies de conciliation d'un horaire de travail variable avec des responsabilités familiales. Le Travail humain, 64, 119-143.

Schwartz, Y. (1994), Travail et philosophie, convocations mutuelles. Octarès, Toulouse.

Six-Touchard, B., Falzon, P. (2013). L'auto-analyse du travail : une ressource pour le développement des compétences. Dans, Ergonomie constructive, ed P. Falzon, pp. 237-249, PUF, Paris.

Six, F., Lancry-Hoestlandt, A., Vaxevanoglou, X. (1991). La conception des ateliers des lycées techniques et des lycées professionnels. GERN : rapport de recherche 88D0773, programme Technologie-Emploi-Travail du M.R.T., 97p.

Teiger, C., Montreuil, S. (1995). Les principaux fondements et apports de l'analyse ergonomique du travail en formation. Education Permanente, 124, 132-138.

Teiger, C., Lacomblez, M. (2006). L'ergonomie et la transformation du travail et/ou des personnes. Permanences et évolutions. Education Permanente, 166, 9-28.

Thin, D. (2002). L'autorité pédagogique en question. Le cas des collèges de quartiers populaires. Revue française de pédagogie, 139.

Thomas-Bion, F., Fiard, J. (2010). Connaissances et croyances juridiques. Le choix des pratiques des enseignants d'éducation physique et sportive du secondaire. Actes du Colloque Analyse des pratiques en EPS. Expériences marquantes et gestes professionnels, Presses universitaires Blaise Pascal, Clermont-Ferrand.

Thomas-Bion, F., Fiard, J. (2008). Choix des pratiques sportives et conscience des risques encourus. Le poids de la loi ressenti par les enseignants d'EPS. Une enquête en région Auvergne. 5 ${ }^{e}$ biennale internationale Association de chercheurs et praticiens dans le domaine de l'intervention en sport ARIS, L'intervention en sport et ses contextes institutionnels : cultures et singularités de l'action, Rodez, 14-16 mai.

Van Zanten, A. (Ed.). (1997). La scolarisation dans les milieux difficiles. INRP- Centre Alain Savary, Paris.

Vors, O., Gal-Petitfaux, N. (2007). Analyse des conditions de la viabilité du travail en classe d'EPS avec des élèves difficiles d'un Réseau ambition réussite. Actes du XII ${ }^{e}$ Congrès international de l'ACAPS Les sciences du mouvement et du sport autour du monde, Leuven, Belgique, 31 octobre, 1-2 novembre, p. 368-370. 


\section{ANNEXES}

\section{Annexe 1}

Codage utilisé dans les graphiques pour les variables de l'ACM (dichotomisées, pour les trois axes)

Variables actives :

echapFacil : la facilité qu'ont les élèves à échapper au cours d'EPS

posTrav : la possibilité de travailler avec sa classe, seul sur un lieu

chargTrav : la charge de travail, et le temps passé au travail

respons : la responsabilité de l'enseignant en cas d'accident, l'accusation possible

d'attouchement en cas de contact corporel lors des parades par exemple

role : la tenue des rôles que l'on est parfois amené à exercer à l'école : enseignant,

éducateur, policier, surveillant

envir : les conditions « environnementales » de l'enseignement (chaleur des gymnases,

froid et vent à l'extérieur en hiver, bruit...)

statut : le statut de professeur aujourd'hui, sa promotion, ses revenus, son pouvoir

d'achat

reconnu : la reconnaissance sociale du professeur d'EPS par les autres enseignants, les

parents d'élèves, les élèves

coopColl : la collaboration des collègues de l'équipe EPS de votre établissement

poidsText : le poids des textes officiels relatifs à l'enseignement de l'EPS en classe et à son

évaluation

chargExt : les charges extérieures à l'enseignement (administration, les diverses

réunions, les bulletins...)

parents : le rôle des parents d'élèves et l'importance que l'école leur donne

Variables supplémentaires :

Au cours des cinq dernières années, ce que l'on appelle globalement votre « forme » s'estelle dégradée?

pleineForme : pas du tout, je suis toujours en pleine forme

qqPbSanté : j'admets de temps à autres quelques problèmes de santé

svtPbSanté : j'ai souvent des problèmes de santé

grosPbSanté : j'ai de gros problèmes de santé

Rentré à votre domicile, après une journée chargée en cours, vous arrive-t-il de vous sentir épuisé, voire de dormir?

epuis_jamais : jamais

epuis_rare : rarement

epuis_freq : fréquemment

epuis_tjrs : tout le temps

Les problèmes professionnels arrivent-ils à troubler votre sommeil ?

somm_jamais : jamais

somm_rare : rarement

somm_freq : fréquemment

somm_tjrs : tout le temps 


\section{NOTES}

1. Nous reviendrons en discussion sur le caractère atypique de l'enquête pour l'ergonomie.

2. Les auteurs se tiennent à disposition pour transmettre le questionnaire utilisé à toute personne intéressée.

3. L'ensemble des résultats relatifs au sexe apparaissent plus loin dans un paragraphe spécifique.

4. Thomas-Bion et Fiard (2010) associent cela notamment à la nécessaire acuité de la surveillance, la mise en jeu de dispositifs de parades pour limiter les chutes et leurs conséquences.

5. La bivalence d'un enseignant correspondrait au fait qu'il enseigne deux matières : l'EPS et une autre.

\section{RÉSUMÉS}

L'enseignement de l'éducation physique et sportive (EPS) dans les collèges et lycées français fait classiquement l'objet de nombreux travaux en didactique et en pédagogie, selon une approche qui privilégie la relation entre l'élève et le savoir. Dans ces travaux, l'activité de l'enseignant n'est généralement interrogée qu'au regard de cette relation qu'il tente de construire. Ainsi de nombreux enjeux de cette activité échappent aux études, alors qu'ils apparaissent largement constitutifs du rapport construit entre l'enseignant et son travail, et donc des formes de mobilisation de cet enseignant dans sa relation à l'élève. L'enquête présentée ici vise précisément à objectiver le rapport au travail des enseignants d'EPS, notamment au travers des relations entre effets sur la santé, perception du métier et conditions de travail. Réalisée en région Auvergne (France) auprès de 218 enseignants d'EPS, les résultats montrent d'abord un écart entre le discours général positif sur le métier et les réponses spécifiques apportées à des questions précises sur l'organisation du travail, le collectif, ou encore la santé, qui révèlent de réelles difficultés. Une corrélation significative apparaît notamment entre certaines difficultés vécues dans le travail relatives aux questions d'organisation du travail, de charge de travail et de sens du travail, et certaines situations de fatigue et d'épuisement. Par ailleurs, des différences liées à l'âge et au sexe sont repérées dans le rapport au travail. Les difficultés soulevées relèvent du champ de l'ergonomie et plusieurs perspectives d'actions peuvent être envisagées : la gestion des fins de carrière, le développement des collectifs et des organisations, l'intervention ergonomique en milieu scolaire, l'intervention ergonomique en conception, la formation continue des enseignants.

Numerous studies in didactics and pedagogy have focused on the relation between the student and knowledge in their examination of how physical education and sports (PES) is taught in French colleges and high schools. In these studies, the only aspect of the teaching activity that is generally examined is how the teacher goes about building this student-knowledge relation. Many of the elements that are part of the teaching activity are consequently ignored, despite the fact that these elements appear to strongly contribute to the links built between the teacher, his work, and his students. Accordingly, the survey presented here aims to objectify the relation of PES teachers to their work, particularly through the relation between health effects, perception of work, and work conditions. Conducted in the Auvergne region of France with 218 PES 
teachers, the results showed that there was a gap between the positive general discourse about work and the specific answers to precise questions about the organization of work, the work collective, and health, these answers revealing real difficulties. A significant correlation appeared between certain difficulties regarding issues of work organization, workload, and the meaning of work on the one hand, and situations of fatigue and exhaustion on the other. Differences related to age and sex were moreover identified. Given that the highlighted difficulties can be addressed by an ergonomics approach, several possible associated actions can be considered: managing end-of-career transitions, developing work collectives and work organizations, conducting ergonomic intervention in teaching organizations and in the early phases of the design project, and taking teachers' needs into account in their training.

La enseñanza de la educación física y el deporte (EPS) en colegios y escuelas secundarias en Francia ha sido objeto de numerosos estudios en didáctica y pedagogía, con un enfoque que enfatiza la relación entre el alumno y el conocimiento. En estos trabajos, la actividad del profesor es generalmente analizada a partir de esta relación que está tratando de construir. De esa manera numerosos elementos importantes de esta actividad escapan al estudio, a pesar de que aparecen como una gran parte constitutiva de la relación construida entre el profesor y su trabajo, y por lo tanto como formas de movilización de este profesor en su relación con los alumnos. El estudio que aquí se presenta se plantea precisamente objetivar la relación de trabajo de los profesores de educación física, especialmente a través de la relación entre los efectos sobre la salud, la percepción de la profesión y las condiciones de trabajo. Llevada a cabo en la región de Auvernia (Francia) a partir de 218 profesores de educación física (EPS), los resultados muestran por primera vez una brecha entre el discurso general positivo sobre la profesión y respuestas específicas a preguntas precisas acerca de la organización del trabajo, el colectivo, o la salud, que revelan dificultades reales. Una correlación significativa aparece entre ciertas dificultades vividas en el trabajo relativas a cuestiones de organización del trabajo, de carga de trabajo y de sentido del trabajo, y algunas situaciones de fatiga y agotamiento. Por otra parte, diferencias relacionadas con la edad y el sexo se identifican en la relación con el trabajo. Las dificultades señaladas entran en el ámbito de la ergonomía y múltiples perspectivas de acciones pueden ser consideradas: gestión de fin de carrera, el desarrollo de colectivos y de organizaciones, la intervención ergonómica en medio escolar, la intervención ergonómica en concepción, la formación continua de los docentes.

INDEX

Palabras claves : salud de los docentes, organización del trabajo, ergonomía

Keywords : teacher's health, work organization, ergonomics

Mots-clés : santé des enseignants, organisation du travail, ergonomie

\section{AUTEURS}

\section{FABIEN COUTAREL}

Clermont Université, Université Blaise Pascal, ACTé

\section{CÉLINE MARDON}

GIS-CREAPT, Centre d'études de l'emploi 


\section{SERGE VOLKOFF}

GIS-CREAPT, Centre d'études de l'emploi

\section{JACQUES FIARD}

Clermont Université, Université Blaise Pascal, ACTé

MICHEL RÉCOPÉ

Clermont Université, Université Blaise Pascal, ACTé

JOFFREY BEAUJOUAN

Clermont Université, Université Blaise Pascal, ACTé

\section{GÉRALDINE RIX}

Clermont Université, Université Blaise Pascal, ACTé 